\title{
Hard Diffraction at Colliders
}

\author{
Rafał Staszewski ${ }^{1, a}$ \\ ${ }^{1}$ Institute of Nuclear Physics Polish Academy of Sciences, PL-31342 Kraków, Poland
}

\begin{abstract}
The phenomenon of hard diffraction is presented. The discussion includes: the present understanding of the process, experimental techniques, past measurements performed at the SPS, HERA and Tevatron colliders, the first LHC data and prospects for new results.
\end{abstract}

\section{Introduction}

Diffractive interaction is a process that is governed by strong forces, but in which no quantum numbers are transferred. The most important types of diffractive processes are shown in Fig. 1. The simplest of them is the elastic scattering, where the final state consists of the same particles as the initial state - both particles stay intact. In single diffractive dissociation (single diffraction), one of the particles remain intact, while the other dissociates into a multi-particle state. In double dissociation (double diffraction), both particles dissociate. Finally, in central diffraction both particles stay intact, but the final state consists also of additional particles.

In the Regge theory diffractive interactions are governed by the exchange of the pomeron trajectory, which intercept is close to unity and which is responsible for the growth of the total cross sections with the centre-of-mass energy.

In the quantum chromodynamics the pomeron can be described in the leading order as an exchange of two gluons in a color-signet state. However, at small $t$ values ( $t$ is the four-momentum transfer squared and the cross section for diffractive processes is steeply falling with $t$ ) the strong coupling constant is not small enough to justify neglecting higher-order diagrams. Therefore, the pomeron should be thought of rather as an exchange of a gluon ladder.

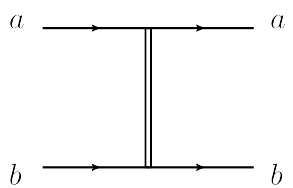

(a)

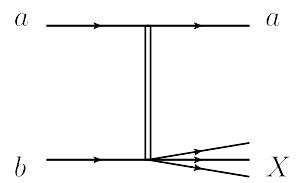

(b)

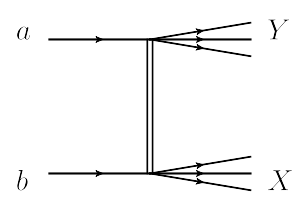

(c)

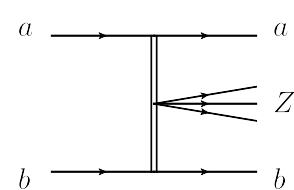

(d)

Figure 1. Diagrams of diffractive processes: (a) elastic scattering, (b) single diffractive dissociation (single diffraction), (c) double diffractive dissociation (double diffraction) and (d) central diffraction.

a e-mail: rafal.staszewski@ifj.edu.pl 


\section{Early history}

The concept of hard diffraction was born from the assumption that one can consider the single diffractive process as a collision of a hadron with a pomeron emitted by the other hadron [1]. If one assumes that the pomeron consists of partons, then the pomeron-hadron interaction resembles any other hadron-hadron collision. In particular, it is possible that the partons undergo a hard interaction, even though the diffractive exchange (emission of the pomeron) is a soft process. Such a process would lead to a signature with an intact hadron and a hard object (e.g. a pair of high- $p_{T}$ jets) present in the dissociated state.

The above hypothesis was studied first at the CERN SPS collider with $p \bar{p}$ collisions at $\sqrt{s}=$ $630 \mathrm{GeV}$. The UA8 experiment measured the intact protons and anti-protons, which are scattered at very small angles, with detectors inserted into the accelerator beam pipe with special mechanisms Roman pots. The jets were detected with the central calorimeter of the UA2 experiment.

The experiment confirmed the existence of hard diffraction [2]. In events with a tagged proton that possessed more than $90 \%$ of its initial energy, localised depositions of energy were observed in the calorimeters. These clusters showed typical properties of jets and were similar to the ones observed in non-diffractive events.

\section{Mechanism of hard diffraction}

Although the hypothesis of existence of hard diffractive events was confirmed, the interpretation in terms of the underlying mechanism was, and still remains, not straightforward. Apart from the picture of the resolved pomeron consisting of partons, other descriptions of hard diffraction are possible.

A hard diffractive interaction can be obtained in a scenario, when the hard object is produced as in any other non-diffractive interaction, but in addition some soft gluon exchanges are presents in the event [3]. These gluons can screen the color-flow present in the hard event. Then, in the full event the overall exchange would be colour-singlet. The process would be considered diffractive, without the need of introducing a pomeron consisting of partons.

\section{Diffractive PDFs}

Detailed experimental studies of hard diffraction were performed at the HERA ep collider. The process of diffractive deeply inelastic scattering (DDIS) is depicted in Fig. 2. It is instructive to discuss the kinematics of this process. The relevant variables are:

- $Q^{2}$ - minus the square of the four-momentum exchanged by the electron,

- $t$ - the square of the four-momentum exchanged by the proton (which stays intact),

- $W$ - invariant mass of the photon-proton system,

- $M_{X}$ - invariant mass of produced particles apart from scattered electron and proton,

- $x$ - fraction of the proton carried by the struck quark,

- $x_{I P}$ (sometimes $\xi$ ) - fraction of the momentum lost by the proton,

- $\beta=x / x_{I P}$ - fraction of the momentum lost by the proton that was carried by the struck quark.

In addition, it is possible to assume a diffractive DIS event in which the proton does not stay intact, but dissociates. Then, additional kinematic variable has to be introduced: $M_{Y}-$ the mass of the dissociated state. 


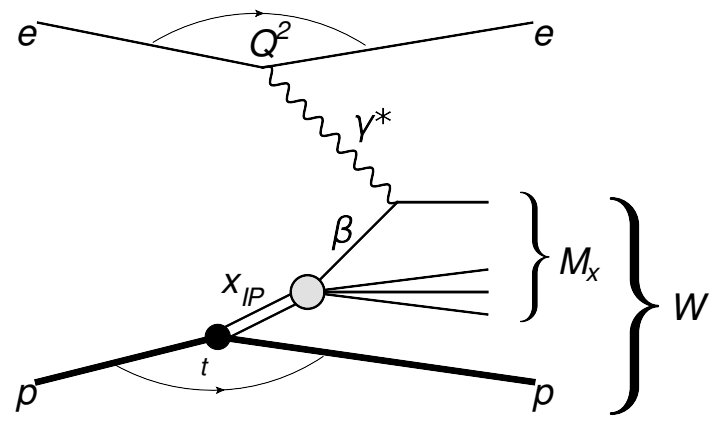

Figure 2. Feynman diagram of diffractive deeply inelastic scattering.

The DDIS process can be thought of as a scattering of the electron off a quark inside a pomeron emitted from the proton. Then, $x_{I P}$ is the fractional momentum carried by the pomeron, $\beta$ is the fraction of the pomeron momentum carried by the quark and $M_{X}$ is the photon-pomeron invariant mass.

Similarly to the case of inclusive DIS, it is possible to measure the diffractive structure functions $F_{2}^{D}$. However, it no longer depends only on $x$ and $Q^{2}$, but also on the proton kinematics: $t$ and $x_{I P}$. Since experimentally it is not always possible to detect the intact proton (or the dissociated state), one can also consider the structure functions averaged over proton kinematics. The common naming convention is: $F_{2}^{D(4)}=F_{2}^{D}\left(x, Q^{2}, x_{I P}, t\right), F_{2}^{D(3)}=\int F_{2}^{D(4)} \mathrm{d} t, F_{2}^{D(2)}=\int F_{2}^{D(3)} \mathrm{d} x_{I P}$.

It has been theoretically shown [4] that for DDIS processes the QCD factorisation holds: the cross section for hard processes can be calculated as convolution of the hard matrix element with parton distributions. Therefore, one can introduce the diffractive PDFs, which also depend on $x, Q^{2}, x_{I P}$ and $t$. In addition, it is often assumed that these distributions factorise into the pomeron flux and its structure:

$$
f_{p}\left(x, Q^{2}, x_{I P}, t\right)=\Phi\left(x_{I P}, t\right) \cdot f_{I P}\left(\beta, Q^{2}\right) .
$$

Assuming the DPDFs undergo a QCD evolution like the inclusive PDFs, the pomeron structure was extracted from the $F_{2}^{D}$ measurements [5].

\section{Factorisation breaking}

The diffractive PDFs measured at HERA can be used to predict hard diffractive cross sections for other processes. Such predictions have been compared to the measurements performed at the Tevatron accelerator. It turned out that the predictions overshot the measurement by about one order of magnitude. This shows that the factorisation theorem regarding diffractive interactions, which has been proven for DDIS, does not hold for other processes, like $p \bar{p}$ collisions.

The physical mechanism of the factorisation breaking comes from the fact that hadrons have finite size, unlike the highly virtual photon in DIS. Therefore, it is possible that in a hadron-hadron collision several independent (soft) interactions take place. If one of them is diffractive, but others are not, the overall event would not have a diffractive signature.

In order to have a diffractive event, the diffractive interaction must not be accompanied by any other soft interaction. Probability of such a case is often called rapidity gap survival probability (the origin of the name will be explained in Section 6). The details of this correction factor, like its energy, kinematics, process dependence, etc. are a subject the present research. 


\section{Experimental techniques}

Two experimental techniques have been employed in measurements of diffraction. The first one, already mentioned in Section 2, is the direct registration (tagging) of the intact hadron that was scattered diffractively. In accelerator experiments this requires dedicated detectors insertable into the beam chamber. Typically this is performed with Roman-pot mechanisms, but also another solution has been used, where a whole section of the beam pipe was movable and the detectors were fixed to it (Hamburg movable beam pipe).

The other technique is based on another property of diffractive events. When a colour singlet is exchanged, the particle production in the rapidity region of the exchange is strongly suppressed compared the exchange of a coloured object. In diffractive events one may more often find large rapidity gaps.

Both above methods have their advantages and disadvantages. The tagging method provides the reconstruction of the scattered hadron momentum, allowing more differential measurements. On the other hand, the kinematic region of the detector acceptance is limited by its position and the magnetic fields of the accelerator. The rapidity-gap method typically uses the already existing calorimetric system of the experiment and does not require dedicated equipment. On the other hand, it does not allow distinguishing between the single-diffractive events and double-diffractive ones, in which one of the dissociation mass is so small that the system escapes the calorimeter acceptance. In addition, this method is limited to large sizes of rapidity gaps, which translates into rather small masses of the dissociated system. High-mass diffraction has gaps of similar size to those present in non-diffractive processes because of statistical fluctuations.

The common experimental problem common for both methods is pile-up - a presence of several independent hadron-hadron interactions in a single registered event, which is a result of high luminosity of current hadron colliders. A rapidity gap present in the diffractive event will be filled with particles originating from another, independent interactions. The higher the pile-up, the more difficult it is to observe events with large gaps.

The presence of pile-up affects the tagging method in a different way. A hard non-diffractive event can be accompanied by a soft single-diffractive process that produces a forward intact hadron, which results in a hard-diffractive signature of the whole event. Here, pile-up becomes a source of background.

Pile-up is a big experimental problem, because it limits the instantaneous luminosity in which diffractive events can be studied. This, in turn, translates into limitations on the values of cross sections for processes that can be measured. This is particularly true for the LHC, where the average pile-up multiplicity is of the order of twenty. Apart for special cases of exclusive events, which will be discussed in the next section, diffraction cannot be measured in such conditions and require dedicated runs.

\section{Variety of diffractive topologies}

As discussed before, diffractive interaction means an exchange of a pomeron. However, this exchange can occur in a number of ways. In the following, different types of diffractive processes will be discussed on the example of the jet production.

If a single pomeron is exchanged leaving one of the hadrons intact, then one deals with the single diffractive jet production. This can be thought of as a collision of the pomeron with the other hadron, which in illustrated in Fig. 3a.

If two pomerons are exchanged and both hadrons stay intact, the process is called central diffraction or a double pomeron exchange and its diagram is presented in Fig. 3b. Fig. 3c presents a similar 


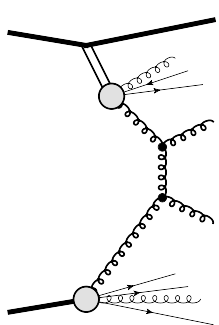

(a)

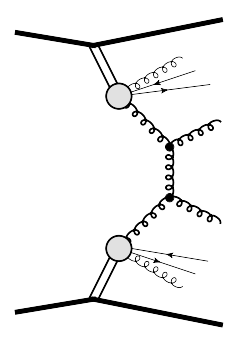

(b)

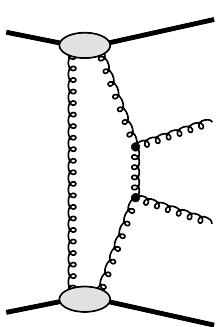

(c)

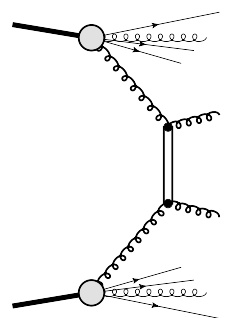

(d)

Figure 3. Different types of diffractive jet production processes: (a) single diffraction, (b) central diffraction, (c) central exclusive production, (d) double diffraction (jet-gap-jet).

process, called central exclusive production, which also contains two intact hadrons and a central di-jet system.

The difference between the exclusive and non-exclusive central diffraction is in the presence of the pomeron remnants. Central diffraction can be thought of a collision of two pomerons consisting of partons. One parton from each of the pomerons participates in the hard process producing the jets. However, other pomeron-originating partons may not interact, or interact softly. They are called pomeron remnants, are produced at small angles and usually escape detection. There are no remnants in the exclusive process - the whole energy taken from the initial-state hadrons goes into the production of the central state.

The exclusive processes are a very special types of hadron-hadron interaction. For them, using the detectors that can measure diffractively scattered hadrons, it is possible to measure all particles of the final state. They are very interesting also from the phenomenological point of view, since they can be calculated directly in QCD, without the need of introducing the diffractive PDFs. The experimental detection of these processes is a challenge because of their very small cross sections. However, the energy-momentum conservation introduces very strong correlations between the centrally produced state and the forward hadrons. This allows their measurement even in pile-up-contaminated environment.

Fig. 3a presents a diagram for the jet-gap-jet process. Here, unlike in all above cases, the pomeron exchange is a part of the hard process. The colour singlet is exchanged between the jets. In this process both hadrons dissociate, so the tagging method cannot be used. The large rapidity gap will be present between the jets.

\section{The Large Hadron Collider}

At the time of writing this article LHC has already provided first measurements on hard diffraction. The diffractive contributions have been observed for the jet and electroweak bosons [6-8]. All these measurements are based on the rapidity gap method and their precision is rather limited.

Two LHC experiments, ATLAS and CMS, are now equipped with systems of detectors that are used for tagging diffractively scattered protons. It is expected that they will provide much more detailed and precise measurements of hard diffraction in various topologies. The forward physics programme for the LHC including feasibility studies for many processes can be found in the report [9] prepared by a dedicated Forward Physics and Diffraction Working Group. 


\section{Acknowledgements}

This work was supported in part by Polish National Science Centre grant 2012/05/B/ST2/02480.

\section{References}

[1] G. Ingelman and P. E. Schlein, Phys. Lett. 152B, 256 (1985). doi:10.1016/0370-2693(85)91181-5

[2] R. Bonino et al. [UA8 Collaboration], Phys. Lett. B 211, 239 (1988). doi:10.1016/03702693(88)90840-4

[3] A. Edin, G. Ingelman and J. Rathsman, Phys. Lett. B 366, 371 (1996) doi:10.1016/03702693(95)01391-1 [hep-ph/9508386].

[4] J. C. Collins, Phys. Rev. D 57, 3051 (1998) Erratum: [Phys. Rev. D 61, 019902 (2000)] doi:10.1103/PhysRevD.61.019902, 10.1103/PhysRevD.57.3051 [hep-ph/9709499].

[5] A. Aktas et al. [H1 Collaboration], Eur. Phys. J. C 48, 715 (2006) doi:10.1140/epjc/s10052-0060035-3 [hep-ex/0606004].

[6] CMS Collaboration, Eur. Phys. J. C 72, 1839 (2012).

[7] CMS Collaboration, Phys. Rev. D 87, no. 1, 012006 (2013).

[8] ATLAS Collaboration, Phys. Lett. B 754, 214 (2016).

[9] K. Akiba et al. 2016 J. Phys. G: Nucl. Part. Phys. 43110201. 